\title{
Por métricas alternativas mais relevantes para a América Latina
}

\author{
More relevant alternative metrics \\ for Latin America
}

Germana BARATA ${ }^{1,2}$ (D) 0000-0001-6064-6952

\section{Resumo}

As métricas alternativas têm sido utilizadas como complemento às métricas tradicionais, funcionando como ferramentas para rastrear os usos e a atenção social a publicações científicas no ambiente online. As revistas científicas de acesso aberto têm, potencialmente, maior chance de serem compartilhadas e lidas pelo público nas redes sociais. Nesse cenário, a produção científica do Brasil e da América Latina, a exemplo daquelas indexadas no SciELO, que reúne revistas científicas relevantes e de acesso aberto da região, poderiam se beneficiar da altmetria. No entanto, análises sobre a representatividade de países, idiomas e áreas do conhecimento na altmetria revelam que é preciso aperfeiçoar os indicadores para que valorizem a ciência publicada por países em desenvolvimento, de língua não inglesa, de acesso aberto e relevância nacional ou regional. Tendo isso como base, este paper aponta limitações da altmetria para a ciência praticada na América Latina, tomando como análise o comportamento de uso de redes sociais de acadêmicos e sociedade em geral, a presença de diferentes idiomas para compartilhar artigos de um mesmo tema, bem como os dados de plataformas que geram dados altmétricos, com enfoque para a provedora de dados inglesa Altmetric.com. A altmetria não tem sido capaz de retratar a atenção que artigos científicos brasileiros recebem nas redes sociais, apesar de haver esforços por parte das revistas em divulgação científica e do relativo engajamento social que conseguem mobilizar. É preciso ampliar a coleta de dados no Facebook, em português e espanhol, além de amplificar as coleções de blogs e veículos jornalísticos na região.

Palavras-chave: Acesso aberto. Altmetria. Comunicação acadêmica. Divulgação científica. Redes sociais.

\begin{abstract}
Alternative metrics have been used as a complement to traditional metrics as tools to track the uses and social attention to scientific publications in the online environment. Open access scientific journals are potentially more likely to be shared and read by the public on social media. In this scenario, the scientific output of Brazil and Latin America, like those indexed in the SciELO database, which brings together relevant and open access scientific journals from the region, could benefit from altmetrics. However, analyzes of the representativeness of countries, languages and fields of knowledge in altmetrics reveal that the indicators need to be improved to value science in developing, non-English speaking, open access countries and national or regional relevance. This paper points out the limitations of altmetrics for science in Latin America, by analyzing the social media behavior of scholars and society, the presence of different languages to share articles, as well as the data that generate altmetrics, such as the ones produced by the British data provider

\footnotetext{
1 Universidade Estadual de Campinas, Núcleo de Desenvolvimento da Criatividade, Laboratório de Estudos Avançados em Jornalismo. R. Seis de Agosto, 50, Cidade Universitária, 13083-873, Campinas, SP, Brasil. Correspondência para/Correspondence to: G. BARATA. E-mail: <germana@unicamp.br>.

2 Simon Fraser University, ScholCommLab. Downtown Vancouver (BC), Canada.

Apoio: Fundação de Amparo à Pesquisa do Estado de São Paulo (Fapesp) (Processos no. 2016/14173-2 e 2018/19870-9).
}

Recebido em 8 de março de 2019, versão final reapresentada em 29 de julho de 2019 e aprovado em 22 de outubro de 2019.
\end{abstract}

Como citar esse artigo/How to cite this article

Barata, G. Por métricas alternativas mais relevantes para a América Latina. Transinformação, v.31, e190031, 2019. http://dx.doi.org/10.1590/23180889201931 e190031 
Altmetric.com. Altmetrics have not been able to portray the attention that Brazilian scientific papers have received in social media, despite the efforts made by journals to disseminate science and engage with the public. It is necessary to expand data collection on Facebook, of contents and channels in Portuguese and Spanish, as well as to expand data collection of blogs and news outlets in the region.

Keywords: Altmetrics. Open access. Scholarly communication. Science communication. Social media.

\section{Introdução}

A divulgação nas redes sociais é hoje uma das principais formas de a informação atingir públicos de forma rápida e massiva. Apesar de haver informalidade das interações que nelas ocorrem, as redes sociais têm sido objeto de pesquisa para entender hábitos de consumo, escolhas eleitorais e tendências de mudanças de comportamento (Eltantawy; Wiest, 2011; Shirky, 2011), incluindo opiniões relacionadas à ciência (Brossard; Schefeule, 2013).

As redes sociais também têm sido a aposta para impulsionar a democratização do conhecimento e o acesso à informação, além de acelerarem a velocidade de disseminação, promoverem mais interações entre acadêmicos e sociedade e provocarem mudanças na comunicação científica (Barata, 2018a).

No Brasil, $67 \%$ da população tem acesso à internet; entre estes, 77\% afirma usar as redes sociais (Comitê Gestor da Internet, 2018), sendo o Facebook a preferida no país e no mundo (Statista, 2019). Em 2018, as projeções chegaram a 271 milhões de usuários no Facebook (Statista, 2018b) e 39.4 milhões no Twitter apenas no Brasil (Statista, 2018c).

As redes sociais geram dados diversos de acesso e compartilhamento de informações científicas; esses dados compõem parte dos indicadores alternativos ou complementares - como preferem os especialistas da área -, para aferir impacto social da ciência (Sugimoto; Larivière, 2018). Mudanças em direção a métricas alternativas foram feitas em 2009, quando a PLoS One estabeleceu a Article-Level metrics (métricas no nível de artigo) para valorizar e medir os usos de artigos a fim de tirar a centralidade da avaliação de revistas, como fazem as métricas tradicionais (vide o fator de impacto). Dentre esses usos, convém mencionar a mensuração do acesso, os downloads, as marcações, os comentários de leitores, as menções em blogs e mídia etc. (Binfield, 2009). Quem cunhou o termo "altmetrics" (em português altmetria) foi Jason Priem por meio de um Tweet (publicação na rede social Twitter) em 28 de setembro de 2010, como preferência em relação a "article-level-metrics." Priem e colegas marcariam o início do debate sobre "altmetria" no Altmetrics: a manifesto (Priem et al., 2010). Posteriormente, a altmetria seria adotada por indexadores (como o Web of Science, Scopus e Scientific Electronic Library Online [SciELO]), revistas científicas ou editoras (Nature, Elsevier) e geraria grande interesse de pesquisa como disciplina emergente (González-Valiente; Pacheco-Mendonça; Arencibia-Jorge, 2016).

A altmetria rastreia os usos sociais de artigos científicos compartilhados em redes sociais e outras plataformas online, como Wikipedia, notícias jornalísticas e blogs, através da identificação do Digital Object Identifier (DOI) ou Application Programming Interface (API) e atribui pontuações distintas a cada um deles (Davies, 2015), compondo o índice altmétrico. Dentre as bases provedoras de dados altmétricos está a inglesa Altmetric.com, Plum X, Impact Story (Nascimento, 2017); cada uma delas rastreia dados e atribui pontuações de atenção social a artigos de forma diferente, o que gera índices altmétricos distintos.

A altmetria dá maior ênfase ao artigo em comparação à ênfase dada à revista, como faz o tradicional Fator de Impacto ou o Qualis Periódicos, que avalia revistas científicas no Brasil. Ademais, vai além de medir o "impacto" entre especialistas, uma vez que inclui em sua avaliação novos atores sociais, fontes de dados e ferramentas, além de tipos de produção acadêmica e de interação (Maricato; Lima, 2017). De forma ampla, a altmetria melhora a visibilidade dos artigos e a heterogeneidade dos indicadores, tirando o enfoque acadêmico das produções (Sugimoto et al., 2017). Gouveia (2016) lembra que a altmetria pode ser um indicador de áreas de interesse social ou controvérsias, portanto o interesse social pode ser distinto do acadêmico, muito embora inúmeras análises 
se voltam para comparações entre as métricas alternativas e os indicadores tradicionais (Maricato; Martins, 2017; González-Valiente et al., 2016).

Por incluir em seus indicadores menções e compartilhamentos de artigos científicos em notícias jornalísticas, blogs e redes sociais, a altmetria valoriza esforços de divulgação científica, de diálogos entre ciência e sociedade. Com isso, ela traz perspectivas interessantes para se medir o quanto os artigos científicos de acesso aberto têm conseguido ultrapassar os muros da academia, sobretudo se for considerado o crescimento da demanda por artigos de acesso aberto no mundo (Piwowar et al., 2017). Levando em consideração que a maior parte da ciência brasileira é realizada por universidades públicas, ou seja, financiada com recursos públicos (Clarivate Analytics, 2018), nesse cenário, os esforços de divulgação científica e os usos da ciência pela sociedade por meio da altmetria são estratégicos, especialmente se forem capazes de medir a atenção social da ciência em níveis nacional e regional.

Alperin (2013) defende que a altmetria tem o potencial de reconhecer e destacar uma ciência mais multinacional e multidisciplinar, como a da América Latina, especialmente se for considerado que os indicadores tradicionais têm beneficiado países desenvolvidos e de língua inglesa. Vanti e Sanz-Casado (2016) também valorizam a democratização da ciência, uma vez que as métricas alternativas podem ser usadas "a favor da produção científica oculta e excluída das principais bases de dados de citação bibliográfica", além de fomentarem "a investigação sobre temas de interesse local e nacional", portanto contribuindo para uma ciência mais democrática. No entanto, alguns estudos têm mostrado que os indicadores altmétricos também priorizam dados relativos à ciência desenvolvida em países desenvolvidos e de língua inglesa (Alperin, 2015; Zahedi; Costas, 2017; Barata, 2018b).

A inclusão de inúmeros fatores que compõem as métricas alternativas configura tanto sua riqueza quanto sua fragilidade. Muitos estudos questionam as limitações da altmetria como indicador de "impacto", falta de clareza sobre o que realmente medem, reflexão teórica fragilizada, dados contraditórios (Sugimoto et al., 2017), entre outros; portanto, claramente é preciso validar a qualidade dos dados produzidos para que a altmetria tenha maior robustez e mais credibilidade (Vanti; Sanz-Casado, 2016).

A América Latina detém 3,5\% dos pesquisadores do mundo (Estenssoro et al., 2016), é responsável por cerca de 4\% dos artigos indexados no Scopus (Noorden, 2014a) e, mais importante, tem sido responsável por um protagonismo nas publicações em acesso aberto (Sánchez-Tarragó et al., 2016). Quanto ao Brasil, embora a pesquisa com altmetria ainda seja incipiente, há sinais de crescimento da comunidade e relevância dos debates sobre o tema, como ficou explícito na primeira conferência dedicada ao tema, que ocorreu no Rio de Janeiro em novembro de 2018, a I LATmetrics: métricas alternativas e ciência aberta da América Latina.

González-Valiente, Pacheco-Mendoza e Arenciba-Jorge (2016) levantaram publicações sobre altmetria indexadas no Web of Science e Scopus e identificaram que a maioria delas tem autores europeus e norte-americanos, com baixa representação da América Latina, Ásia e África. Na América Latina, uma simples busca feita em uma das bases de indexação mais relevantes para a região, o SciELO, mostra a existência de 13 artigos de 2014 a 2018, dos quais sete são de autores do Brasil, três da Colômbia, um da Argentina, um de Cuba e um do Peru. A busca por artigos foi feita com a palavra-chave "altmetr*" para todos as categorias, no dia 30 de janeiro de 2019.

Para o caminho da internacionalização, o Brasil não pode ficar à margem das pesquisas sobre altmetria, principalmente para garantir que se valorizem as particularidades do país e da região, como os usos acadêmicos das redes sociais.

Tendo as questões acima como base, este artigo analisa as limitações da altmetria que impedem a visibilidade da ciência realizada no Brasil como exemplo de país de boa produtividade científica na América Latina, apesar de ter fortalecido as práticas de divulgação científica nas redes sociais. O objetivo é contribuir para aperfeiçoar os usos dos indicadores alternativos na valorização da ciência em nível nacional e regional. Toma-se por base estudos e análises conduzidas por inúmeros autores com enfoque, principalmente, nas métricas alternativas produzidas pela Altmetric.com. 
As perguntas que conduzem este artigo são: Quais são os entraves que impedem a altmetria de identificar os usos sociais de artigos do Brasil e da América Latina? Como tornar a ciência nacional e regional relevante para as métricas alternativas?

\section{A comunidade acadêmica e artigos brasileiros nas redes sociais}

Pesquisa desenvolvida por Barata et al. (2018a) analisou os usos de diferentes redes sociais por acadêmicos de duas universidades brasileiras, sendo elas a Universidade Estadual de Campinas (Unicamp) e a Universidade Federal de Alagoas (UFAL). O questionário, distribuído em 2017 por mailing-list para 7.356 professores e pesquisadores, com taxa de resposta de 10,0\%, revelou que há usos que variam de acordo com as áreas do conhecimento, mas com padrão semelhante entre as universidades. Dentre as três redes sociais usadas de modo profissional com maior frequência, o destaque foi para o WhatsApp, preferido por 61,6\% dos respondentes, seguido pelo ResearchGate (54,1\%), e Facebook (47,7\%), enquanto 8,8\% escolheram o Twitter.

Esse resultado indica como se deve olhar para as pesquisas internacionais com zelo, pois elas querem indicar uma tendência internacional de uso homogêneo das redes sociais por acadêmicos, como sugere Noorden (2014b) em análise feita com usuários da ResearchGate. Igualmente, as pesquisas em altmetria dão destaque a análises com dados do Twitter (Haustein, 2018), enquanto pesquisadores brasileiros e, provavelmente, latino-americanos (se comparados às tendências de usos da população), utilizam com maior frequência o Facebook em relação ao Twitter, como ocorre também no comportamento da população em geral (Statista, 2018a).

Alperin (2015) concluiu que, dentre os 389.795 artigos publicados de 2000 a 2014 no SciELO, cerca de 25,0\% deles recebiam alguma pontuação do Altmetric - o chamado Altmetric Attention Score - bem abaixo de coberturas encontradas de artigos da PubMed, Web of Science (WoS) e arXiv, por exemplo. Dentre os artigos que receberam alguma atenção nas redes sociais, houve grande diferença da atenção recebida entre as métricas de redes sociais, visto que, em apenas três delas, os artigos SciELO receberam uma atenção significativa, embora também considerada baixa: Mendeley (18,8\%), Twitter (6,0\%) e Facebook (2,8\%). Entre as possíveis explicações para a baixa cobertura dos artigos no Altmetric, Alperin sugeriu o baixo uso de artigos nas redes sociais, o baixo uso de redes sociais por acadêmicos na região ou uma cultura diferente para compartilhar publicações científicas.

Outra análise sobre a performance dos top-100 artigos do SciELO com maior pontuação altmétrica no Facebook e Twitter em abril de 2016 (Maricato; Lima, 2017) identificaram pontuação média bastante baixa por artigo (9,41), com destaque para a área de Ciências da Saúde (43,0\% do total de artigos). Para cada postagem nas redes sociais, os autores classificaram impacto acadêmico (IA) ou social (IS), de acordo com o perfil do público que compartilhava o artigo. Eles concluíram que o IA foi muito superior em ambas as redes sociais, mas no Facebook há maior potencial de gerar IS.

Araujo e Alves (2018) avaliaram a performance no Altmetric de artigos de bolsistas produtividade Conselho Nacional de Desenvolvimento Científico e Tecnológico (CNPq) no currículo Lattes, considerados um grupo acadêmico de prestígio e alta produtividade no País. A pontuação no Altmetric (ou Altmetric Attention Score) foi muito baixa, inclusive para o Facebook - rede social mais usada no Brasil; houve resultado mais significativo no Mendeley e no Twitter, para artigos em inglês e artigos referentes às áreas das Ciências da Saúde e Biológicas. O resultado levou os autores a considerarem que falta uma cultura de compartilhamento de artigos em redes sociais, além de haver limitação nos dados de cobertura altmétrica para a América Latina.

Fraumann et al. (2016) constataram que os artigos publicados em 296 revistas brasileiras indexadas no SciELO, de 2012 a 2015, registraram apenas cinco indicadores de altmetria significativos, embora ainda tenham gerado baixa pontuação no Altmetric Attention Score: Twitter, Facebook, blogs, notícias e citações no Wikipedia. Os autores compararam a atenção social que artigos receberam no Twitter entre revistas que possuem e revistas que 
não possuem conta nessa rede social, concluindo que os artigos das primeiras recebem um pouco mais de atenção no Twitter. Há ainda vantagem para as revistas com mais de 180 seguidores que geraram mais atenção também no Facebook e blogs. No entanto, pode-se argumentar que o número de revistas com contas no Twitter é ainda muito baixo (Barata; Medeiros; Kishi, 2016), o que também reflete o desinteresse dos brasileiros em relação a essa rede social.

Uribe-Tirado e Alhuay-Quispe (2017) também encontraram um resultado bastante interessante para os 55 autores mais produtivos da ibero-américa na área de alfabetização informacional, ou seja, aqueles profissionais responsáveis por melhorar a conscientização e prática entre os profissionais de bibliometria. Houve maior presença de perfis de autores no ResearchGate (58\%), Facebook (55\%), Academia.edu (51\%) e Google Scholar (49\%) em comparação ao Mendeley (25\%) ou ao Twitter (20\%). O Facebook foi a rede social na qual há o maior número de seguidores, portanto, onde se estabelecem mais relações sociais. Para os autores, há uma correspondência de autores produtivos com a visibilidade deles na Internet, o que permite concluir que bibliometria e altmetria se impactam positivamente.

Os estudos mencionados anteriormente denotam, portanto, existir uma contradição entre uso do Facebook por comunidades acadêmicas e a visibilidade que os artigos que elas publicam nessa rede social, de acordo com os dados rastreados pelo Altmetric.com, provedor de dados altmétricos mais usado por indexadores, periódicos e mais pesquisado por estudiosos da área.

\section{Uso de redes sociais por revistas brasileiras}

Revistas científicas estrangeiras de prestígio, sobretudo as de acesso restrito, têm investido em estratégias de divulgação e marketing científico para ampliar sua visibilidade entre acadêmicos e o público em geral, além de manter seu status e influenciar as políticas de Ciência e Tecnologia (C\&T) (Barata, 2010).

No caso das revistas científicas brasileiras, o interesse pelas redes sociais tem crescido desde 2014, motivadas pela orientação do maior indexador de revistas científicas brasileiras, o SciELO, que determinou como novo critério de inclusão a divulgação e marketing dos artigos publicados em redes sociais e/ou blogs a partir de julho de 2014 (Scientific Electronic Library Online, 2017). O projeto que atuou com revistas científicas brasileiras do Portal de Periódicos da Universidade de São Paulo (USP) de 2013 a 2015 identificou que, dentre as 139 revistas, 36,0\% possuíam página no Facebook, porém apenas 19 revistas (13,7\%) mantinham páginas ativas; o número de contas no Twitter, contudo, era ínfimo (Barata; Medeiros; Kishi, 2016). O projeto produziu notícias, press releases ${ }^{2}$, entrevistas ou postagens a partir de artigos de 114 revistas científicas, divulgadas em diversos veículos de comunicação, e atuou diretamente com editores científicos com um esforço de valorizar e capacitar as equipes para estratégias de divulgação científica nas redes sociais, motivados pela determinação do SciELO.

Outros autores analisaram o uso de redes sociais por revistas científicas brasileiras. Souza et al. (2015) verificaram que, na área de Biblioteconomia e Ciências da Informação, dentre as 15 publicações classificadas nos estratos A1, A2, B1 e B2 do Qualis Periódicos da Capes, apenas duas possuíam contas no Facebook. O mesmo foi detectado por Horimi e Zaninelli (2017) para as revistas da mesma área, embora o número de contas no Facebook já estivesse ampliado. Das 53 revistas amostradas, 11 possuíam contas na rede social, embora apenas 6 delas fossem consideradas ativas e com engajamento social. Apesar de constatarem o potencial de ampliar a divulgação das publicações e interação com o público leito, os autores não avaliaram a qualidade e as estratégias usadas na divulgação feita no Facebook pelas revistas da amostra.

A revista História, Ciências, Saúde - Manguinhos (HCSM) foi uma das pioneiras nos esforços de divulgação nas redes sociais, inaugurando blog e página no Facebook ainda em 2013. Os editores reconheceram que a

\footnotetext{
2 Press releases são materiais de divulgação voltados para jornalistas e costumam servir de base para pautas jornalísticas.
} 
iniciativa mudou a rotina da equipe editorial, interferindo no ritmo de trabalho, custos financeiros e na demanda por novas competências na equipe (Benchimol et al. 2014). Com situação financeira mais estável, a HCSM tem conseguido investir na divulgação em redes sociais em português, espanhol e inglês, também como estratégia de internacionalização, constatando benefícios em sua visibilidade e no diálogo com o público leitor (Kishi, 2017). Porém, é clara a frustração da equipe da HCSM em relação à tímida visibilidade nas redes sociais, segundo a pontuação altmétrica relativa aos esforços de divulgação empregados no Twitter, Facebook, blog e à interação com o público constatada nessas redes sociais (Kishi, 2017). A HCSM também foi objeto de análise de Lemle (2018), que concluiu que o aumento de citações e acessos aos artigos da revista não podem ser atribuídos à divulgação nas redes sociais, embora haja exemplos de picos de acessos a artigos posteriores a sua divulgação no blog e página do Facebook.

Quatro revistas brasileiras da área de saúde pública foram analisadas por Reis (2018) que constatou haver interesse e reconhecimento sobre a importância das redes sociais, sobretudo no Facebook, a rede mais usada. No entanto, a autora conclui que as equipes editoriais ainda não exploram bem os recursos disponíveis nas redes sociais; sendo assim, deveriam investir na profissionalização das estratégias de comunicação para que as postagens gerassem mais engajamento com o público leitor. As dificuldades financeiras, humanas e de infraestrutura foram apontadas pelos editores como os maiores obstáculos para o efetivo uso das redes sociais.

As atividades de divulgação não são vistas como prioritárias para o desenvolvimento de um trabalho professional de revistas científicas nas redes sociais, como no Facebook e Twitter, duas das mais usadas entre equipes editoriais de periódicos brasileiros (Kishi, 2017; Reis, 2018).

\section{As limitações da altmetria}

Apesar de grande uso do Facebook por editores de revistas científicas brasileiras, como mencionado na seção anterior, há importantes limitações para o uso de dados dessa rede social para gerar dados altmétricos, sendo focalizado neste artigo o provedor Altmetric.com, mais usado e analisado entre especialistas em altmetria. Análise de Enkhbayar e Alperin (2018) sugere que os dados que o Altmetric.com captura do Facebook são inferiores à atividade que ocorre na rede social para compartilhar artigos científicos, como mostram dados coletados a partir do API do Facebook. É fato que a empresa britânica que produz esses indicadores alternativos, a Altmetric.com, apenas rastreia o compartilhamento de links de artigos científicos em postagens públicas de grupos ou instituições. Portanto, a rica atividade que ocorre entre usuários do Facebook, mesmo quando publicada em postagens públicas, não é contabilizada.

Artigos de autores da América Latina, como o Brasil, que utilizam o Facebook de forma ativa, apresentarão baixa performance altmétrica se forem considerados apenas os indicadores produzidos a partir da referida rede social. Parte dessa invisibilidade de artigos se dá por problemas de múltiplos DOls ou links de acesso a esses artigos; links quebrados ou com problemas, e que impedem sua identificação, também explicam a referida invisibilidade.

A diferença de ações nas redes sociais versus performance na altmetria também diagnosticou problemas de estratégias de comunicação no blog do SciELO, cujo objetivo é justamente divulgar artigos da coleção para especialistas e público amplo. Uma análise da divulgação realizada na página do SciELO no Facebook durante 2017 revelou que, dentre os 176 artigos de 49 revistas científicas divulgados pelo blog, 69.7\% deles não pontuaram no Altmetric Attention Score (índice altmétrico), 14.3\% pontuaram apenas uma vez, e os demais pontuaram mais de uma vez (Barata, 2017). Considerando que uma única menção em blog rastreada pelo Altmetric.com soma 5 pontos (Altmetric, 2019), há, ao menos, 84\% dos artigos divulgados no blog do SciELO via Facebook que sequer são rastreados pela plataforma, o que impede a pontuação. Parte desse resultado se deu porque as páginas de Press release e Perspectiva: Humanas do blog SciELO (ambas com endereços distintos da página principal do blog), responsáveis pela divulgação de artigos científicos, não estavam sendo rastreadas pela empresa britânica. Parte das 
postagens que divulgava artigos no Facebook incluia links para o Blog, não para os artigos, o que explica a razão de não gerarem pontuação. Há ainda 6,5\% de links DOI dos artigos divulgados que estão errados, não gerando, portanto, indicadores altmétricos.

Outra limitação é o rastreamento de artigos de língua não inglesa, cuja cobertura tem desvantagem. Uma análise realizada com artigos sobre o Zika Vírus compartilhados no Twitter e Facebook buscou entender como um tema de relevância nacional e internacional, durante o primeiro semestre de 2016, quando a Zika foi considerada emergência internacional, foi compartilhado pela sociedade (Barata; Shores; Alperin, 2018). O objetivo era analisar o idioma usado nas redes sociais para compartilhar artigos também em idiomas distintos.

Os resultados indicaram que o inglês é o principal idioma das postagens de artigos, também majoritariamente publicados em inglês, corroborando com análises comparativas de indicadores alternativos entre artigos da Espanha e do Reino Unido (Mas-Bleda; Thewall, 2016). No entanto, no Facebook 24\% das postagens foram em idiomas distintos do inglês, com maioria em português e espanhol, línguas das populações mais atingidas pela epidemia de Zika. Os artigos de autores pertencentes a países que não falam inglês têm mais chance de gerarem postagens em língua não inglesa. Esse resultado indica que é preciso investir na divulgação estratégica do Facebook, se editores e autores de revistas científicas quiserem se comunicar com as populações mais atingidas por doenças, como no caso da Zika em 2016, em que Brasil e América Latina e Caribe foram os mais atingidos, garantindo que o conhecimento científico não fique restrito à comunidade acadêmica.

As redes sociais não são as únicas métricas que apresentam limitações para rastrear a atenção social de artigos científicos. Os blogs são os indicadores cuja pontuação é alta (5), só perdem para notícias jornalísticas (8) (Altmetric, 2019). Um levantamento de dados no Altmetric.com identificou a qualidade de blogs da coleção do Altmetric.com em que há 11 mil blogs curados manualmente (Barata, 2018b). Os dados incluíram 1.898 blogs que divulgaram artigos de autores canadenses no Web of Science e que pontuaram no Altmetric entre 2015 e 2016. Entre os 100 blogs que compartilharam 50.75\% dos artigos, há websites que são listas de artigos científicos, sites que reproduzem press releases de universidades e instituições de pesquisa, sites de clínicas, laboratórios médicos ou farmacêuticos, além de sites de notícias jornalísticas tradicionais, editoras ou revistas científicas. Blogs são definidos como páginas que tradicionalmente compartilham conteúdos originais, de modo informal e cuja comunicação pressupõe engajamento com o público leitor (Wilcox; Brookshire; Goldman, 2016). Entende-se, assim, que os dados referentes a blogs do Altmetric são compostos por páginas que não são blogs; ademais, fazem marketing científico e criam uma falsa percepção sobre a atenção social que os artigos científicos estariam produzindo. Uma análise semelhante para artigos brasileiros ou latino-americanos poderia trazer outras limitações, que não foram objeto de investigação desta análise.

A coleção de blogs para rastreamento de métricas alternativas pelo Altmetric.com é feita através de curadoria manual e, depende, pois, de sugestões para inclusão. Não seria exagero afirmar que há um número superior de blogs em língua inglesa, e que tendem a dar mais atenção a artigos também em língua inglesa.

\section{Discussão}

Trabalhar com redes sociais é trabalhar com limitações. Portanto, fazer uso da altmetria (como também ocorre nas métricas tradicionais) é trabalhar com muitas deficiências. As ferramentas ainda são novas e, por serem as redes sociais pertencentes a empresas, o acesso aos dados é bastante restrito e confuso. O Twitter, por lidar com postagens públicas, em sua maioria, ainda fornece maior volume de dados cujo acesso é simples e relativamente detalhado. Não por acaso, é a rede mais pesquisada entre especialistas de altmetria (Haustein, 2018). No entanto, o Facebook, rede social mais importante em alcance na maior parte do mundo, mas sobretudo no Brasil, tem limitações de acesso aos dados, mesmo considerando as postagens públicas. Por essa razão, o Altmetric coleta 
dados bastante incompletos no Facebook que dão a impressão de que a rede é pouco relevante para a atenção social de informações científicas, sendo vista, portanto, como de pouco valor por acadêmicos do mundo todo. A identificação de métodos alternativos, como a análise de artigos compartilhados em postagens públicas do API do Facebook (Enkhbayar; Alperin, 2018), traz algumas possibilidades interessantes de pesquisa futura.

Uma vez que os dados do Altmetric são padronizados para todos os artigos rastreados, acabam produzindo uma distorção para artigos de diferentes países, idiomas e áreas do conhecimento. Com isso, os dados de altmetria, a exemplo dos divulgados pelo Altmetric.com, privilegiam (como já ocorre com as métricas tradicionais) artigos científicos publicados e compartilhados em países desenvolvidos de língua inglesa (Alperin, 2013; Mas-Bleda; Thelwall, 2016). A altmetria precisa desenvolver indicadores que possam também dimensionar o impacto social da ciência localmente, tema de pesquisas futuras. Portanto, ao invés de funcionar como um indicador complementar às métricas tradicionais e valorizar a ciência periférica publicada em outros idiomas, como defendera Alperin (2013), a altmetria medida por meio do Altmetric.com também contribui para a invisibilidade quanto à produção científica de países do Hemisfério Sul (como o Brasil) da mesma forma que ocorre para as métricas tradicionais.

No Brasil, a atividade no Facebook por acadêmicos e sociedade em geral não está representada, tampouco rastreada pelo Altmetric.com, sobretudo por este ser limitado e não capturar a principal atividade social da rede, aquela existente entre pessoas. Por outro lado, o Twitter, cujos dados são amplamente captados, não possui grande adesão pelos brasileiros ou latino-americanos, incluindo os acadêmicos. É preciso, portanto, aperfeiçoar métodos de análise dos usos de artigos científicos no Facebook, ao invés de incentivar uma mudança no comportamento de uso de redes sociais.

Quanto aos blogs, a análise identificou que a coleção do Altmetric.com é bastante falha e muito forte para blogs norte-americanos. Outro limitante nos dados altmétricos é a falta de conhecimento sobre como os links dos artigos científicos devem ser compartilhados nas redes sociais para que ele seja rastreado pelo Altmetric.

Incluir outros indicadores de redes sociais, como no amplamente usado WhatsApp, e aprimorar dados coletados no YouTube - segunda rede social mais usada no mundo (Statista, 2019) -, blogs e sites de notícias é necessário para que seja multiplicado o rastreamento da atenção social que a ciência tem recebido no ambiente online. Embora plataformas como o Altmetric.com colete dados de inúmeras redes sociais, há a necessidade de se ampliar as coleções de sites de notícias, canais do YouTube e blogs a partir dos quais os dados altmétricos são gerados, de modo a aumentar a representatividade de plataformas de língua não inglesa e de países em desenvolvimento.

Apesar de haver potencial para indicar a visibilidade de artigos científicos, a altmetria ainda não é capaz de identificar a relevância social em nível local ou nacional, enfraquecendo, portanto, a relevância de seu uso em países de língua não inglesa, como o Brasil, e outros latino-americanos. Apesar disso, é importante enfatizar a necessidade de uso moderado e relativizado da altmetria de modo a evitar que se conclua, erroneamente, que a ciência nacional ou regional não tem sido capaz de gerar atenção social.

É preciso valorizar a atividade de divulgação científica, sobretudo o diálogo e o acesso da sociedade aos resultados científicos, construindo políticas científicas que valorizem essas atividades na progressão de carreiras e avaliação de projetos de pesquisa, como algumas agências de fomento já cobram de pesquisadores, a exemplo de projetos de grande porte da Fundação de Amparo à Pesquisa do Estado de São Paulo (Fapesp) (projetos temáticos) e do CNPq (Institutos Nacionais de Ciência e Tecnologia). "O maior desafio para a adoção e uso das métricas alternativas no Brasil é a institucionalização das novas práticas de pesquisa online pelos diversos atores da comunicação científica" (Nascimento, 2017, p.72). Caso contrário, os indicadores altmétricos reproduzirão as limitações de indicadores tradicionais, e visibilidade do Brasil e de países latino americanos ainda será tímida. 


\section{Referências}

Alperin, J.P. Ask not what altmetrics can do for you, but what altmetrics can do for developing countries. Bulletin of the Association for Information Science and Technology, v.39 n.4, p.18-21, 2013.

Alperin, J.P. Geographic variation in social media metrics: an analysis of Latin American journal articles. Aslib Journal of Information Management, v.67, n.3, p.289-304, 2015.

Altmetric. How is the Altmetric Attention Score Calculated? Altmetric Support online. 2019. Available from: https://help. altmetric.com/support/solutions/ articles/6000060969-howis-the-altmetric-attention-score-calculated. Cited: Jul. 12, 2019.

Araujo, R.F.; Alves, M. The altmetric performance of publications authored by Brazilian researchers: Analysis of CNPq productivity scholarship holders. arXiv:1807.06366, 2018.

Barata, G. Nature e Science: mudanças na comunicação da ciência e a contribuição da ciência brasileira (1936-2009). 2010. Tese (Doutorado em História) - Universidade de São Paulo, São Paulo, 2010.

Barata, G. Espaço para melhorar a divulgação de revistas científicas SciELO-Brasil. Blog Ciência em Revista, 9 de novembro de 2017. Disponível em: https://www.blogs. unicamp.br/cienciaemrevista/2017/11/09/scielo-no-facebook/. Acesso em: 20 fev. 2019.

Barata, G. Transformações na comunicação científica tradicional por meio de tecnologias digitais. In: Di Giulio, G.M.; Monteiro, M.S.A. (Org.) Comunicação, política e representação: interfaces com ciência, tecnologia e ambiente. São Paulo: Hucitec, 2018a. p.377.

Barata, G. The need to improve blogs qualitative data on Altmetric. In: Altmetrics18 Workshop, Altmetric Conference 5:AM, 2018. Paper [...]. London, 2018b. Available from: http:// altmetrics.org/altmetrics18/. Cited Jul.10, 2019.

Barata, G. et al. O uso de mídias sociais por acadêmicos brasileiros. In: Encontro Brasileiro de Bibliometria e Cientometria, 6., 2018, Rio de Janeiro. Rio de Janeiro: UFRJ, 2018. p.209-217. Disponível em: https://ebbc.inf.br/ebbc6/docs/6 EBBC2018v2018_07_27.pdf. Acesso em: 1 nov. 2019.

Barata, G.; Medeiros, C.; Kishi, K. Social Impact of the Brazilian Science production through science communication. In: Public Communication of Science and Technology Conference, 14th, 2016, Istanbul, Turkey, 2016. Proceedings [...]. Istanbul, Turkey: PCST, 2016. Available from: https://www. pcst.co/archive/2016. Cited: Nov. 1, 2019.

Barata, G; Shores, K.; Alperin, J.P. Local chatter or international buzz? Language differences on posts about Zika research on Twitter and Facebook. Plos One, v.13, n.1, e0190482, 2018.

Benchimol, J.; Cerqueira, R.; Papi, C. Desafios aos editores da área de humanidadesno periodismo científico e nas redes sociais: reflexões e experiências. Educação e Pesquisa, v.40, n.2, p.347-364, 2014.

Binfield, P. PLoS One: Background, future development, and article-level metrics. In: International Conference on Electronic Publishing, 13th., 2009, Milano, Italy. Proceedings [...]. Milano, Italy: ELPUB, 2009. p. 69-86.
Brossard, D.; Scheufele, D.A. Science, new media, and the public. Science, v.339, n.6115, p.40-41, 2013.

Clarivate Analytics. Research in Brazil: A report for Capes by Clarivate Analytics. Philadelphia: Clarivate Analytics, 2018. Available from: http://www.capes.gov.br/images/stories/ download/diversos/17012018-CAPES-InCitesReport-Final. pdf. Cited: Feb. 19, 2019.

Comitê Gestor da Internet. Pesquisa TIC Domicílios 2018. Available from: https://cetic.br/pesquisa/domicilios/indicadores. Acesso em: 20 fev. 2019.

Davies, F. Numbers behind numbers: the altmetric attention score and sources explained. Altmetric.com blog post, 2015. Disponível em: https://www.altmetric.com/blog/score anddonut/. Cited: Feb 20, 2019.

Eltantawy, N.; Wiest, J.B. Social media in the Egyptian Revolution: Reconsidering resource mobilization theory International Journal of Communication, v.5, p.1207-1224, 2011.

Enkhbayar, A.; Alperin, J.P. Challenges of capturing engagement on Facebook for Altmetrics. arXiv:1809.01194v1, 2018.

Estenssoro, E.; Friedman, G.; Hernández, G. Research in Latin America: Opportunities and challenges. Intensive Care Medicine, v.42, n.6, p.1045-1047, 2016.

Fraumann, G. et al. Twitter presence and altmetrics counts of SciELO Brazil Journals. In: Altmetrics Workshop, 2016, Bucharest. Proceedings... [...]. Bucharest, 2016.

González-Valiente, C.L.; Pacheco-Mendoza, J.; Arencibia-Jorge, R. A review of altmetrics as an emerging discipline for research evaluation. Learning Publishing, v.29, n.4, p.229-238, 2016.

Gouveia, F.C. A altmetria e a interface entre a ciência e a sociedade. Trabalho, Educação e Saúde, v.14, n.3, p.643-645, 2016.

Haustein, S. Twitter in scholarly communication. Altmetric Blog. Londres, 12 June 2018. Available from: https://www. altmetric.com/blog/twitter-in-scholarly-communication/. Cited: Feb. 19, 2019.

Horimi, D.; Zaninelli, T. A utilização do Facebook como ferramenta para divulgação de revistas científicas da ciência da informação. In: Colóquio de Organização, Acesso, Apropriação da Informação e do Conhecimento, 2, 2017, Paraná. Anais [...]. Paraná: Universidade Estadual de Londrina, 2017.

Kishi, K. Caminhos para a visibilidade internacional: um estudo de caso sobre as estratégias de divulgação de três periódicos brasileiros de Ciências Humanas. 2017. Dissertação (Mestrado em Divulgação Científica e Cultural) - Universidade Estadual de Campinas, Campinas, 2017.

Lemle, M.M. Para pares e "impares": a experiência da revista História, Ciências, Saúde - Manguinhos no Facebook. 2018. Dissertação (Mestrado em Divulgação da Ciência, Tecnologia e Saúde) - Casa de Oswaldo Cruz/Fiocruz, Rio de Janeiro, 2018.

Maricato, J.M.; Lima, E.L.M. Impactos da altmetria: aspectos observados com análises de perfis no Facebook e Twitter. Informação e Sociedade, v.27, n.1, p.137-145, 2017. 
Mas-Bleda, A.; Thelwall, M. Can alternative indicators overcome language biases in citation counts? A comparison of Spanish and UK research. Scientometrics, v.109, n.3, p.2007-2030, 2016.

Nascimento, A.G. Altmetria para bibliotecários: guia prático de métricas alternativas para avaliação da produção científica. São Paulo: Scortecci Editora, 2017.

Noorden, R. Van. The impact gap: South America by the numbers. Nature, v.510, n.7504, p.202-203, 2014 a.

Noorden, R. Van. Online collaboration: Scientists and the social network. Nature, v.512, n.7513, p.126-129, 2014 b.

Piwowar, H. et al. The State of OA: A large-scale analysis of the prevalence and impact of Open Access articles. PeerJ Preprints, 5:e3119v1, 2017. Doi: http://dx.doi.org/10.7287/ peerj.preprints.3119v1

Priem, J. et al. Altmetrics: A manifesto, 26 October 2010. Available from: http://altmetrics.org/manifesto. Cited: Feb. 18, 2019.

Reis, M.T.V. O conhecimento científico no Facebook: revistas brasileiras de saúde pública e as interações com seus seguidores. 2018. Dissertação (Mestrado em Saúde Pública) - Universidade de São Paulo, São Paulo, 2018.

Sánchez-Tarragó, N. et al. Las revistas científicas en América Latina hacia el caminho del acceso aberto: un diagnóstico de políticas y estratégias editoriales. Trans/nformação, v.28, n.2, p.152-172, 2016. Doi: http://dx.doi.org/10.1590/2318-088 92016000200003

Scientific Electronic Library Online. Critérios, política e procedimento para admissão e a permanência de periódicos científicos a Coleção SciELO Brasil. São Paulo: SciELO, 2017. Disponível em: http://www.scielo.br/avaliacao/Criterios SciELO_Brasil_versao_revisada_atualizada_outubro_2017 1206.pdf. Acesso em: 1 nov, 2019.

Shirky, C. The political power of social media: Technology, the public sphere and political change. Foreign Affairs, v.90, n.1,p.28-41, 2011.

Souza, U.J. et al. O uso das redes sociais pelos periódicos brasileiros de biblioteconomia e ciência da informação. Revista ACB: Biblioteconomia em Santa Catarina, v.20, n.3, p.584-591, 2015.
Statista. Most popular social networking worldwide as of January 2019, ranked by number of active users (in millions), 2019. The Statistics Portal. Available from: https://www statista.com/statistics/272014/global-social-networksranked-by-number-of-users/. Cited: Feb. 25, 2019.

Statista. Most popular social networking platforms in Latin America as of December 2015, by unique visitors (in millions), 2018a. The Statistics Portal. Available from: https://www. statista.com/statistics/202372/latin-america--largest-socialnetworking-sites/. Cited: Feb. 19, 2019.

Statista. Number of Facebook users in Latin America from 2014 to 2019 (in millions). 2018b. The Statistics Portal. Available from: https://www.statista.com/statistics/282350/numberof-facebook-users-in-latin-america/. Cited: Feb. 19, 2019.

Statista. Twitter user penetration in Latin America from 2012 to 2018. 2018c. The Statistics Portal. Available from: https:// www.statista.com/statistics/304131/twitter-users-latinamerica-penetration/. Cited: Feb. 19, 2019.

Sugimoto, C.R.; Larivière, V. Measuring Research: what everyone needs to know. New York: Oxford University Press, 2018.

Sugimoto, C.R.; Work, S.; Larivière, V.; Haustein, S. Scholarly use of social media and altmetrics: A review of the literature. Journal of the Association for Information Science and Technology, v.68, n.9, p.2037-2062, 2017.

Uribe-Tirado, A.; Alhuay-Quispe, J. Estudio métrico de ALFIN en Iberoamérica: De la bibliometría a las altmetrics. Revista Española de Documentación Científica, v.30, n.3, 2017, e180.

Vanti, N.; Sanz-Casado, E. Altmetria: a métrica social a serviço de uma ciência mais democrática. Trans/nformação, v.28, n.3, p.349-358, 2016.

Wilcox, C.; Brookshire, B.; Goldman, J.G. Science blogging: The essential guide. New Haven: Yale University Press, 2016. p.2882016.

Zahedi, Z;; Costas, R. How visible are the research of different countries on WoS and Twitter? An analysis of global vs. Local reach of WoS publications on Twitter. In: International Conference on Scientometrics \& Informetrics (ISSI), 16., 2017, Wuhan. Proceedings [...]. Wuhan, 2017. 